Historic, Archive Document

Do not assume content reflects current scientific knowledge, policies, or practices. 



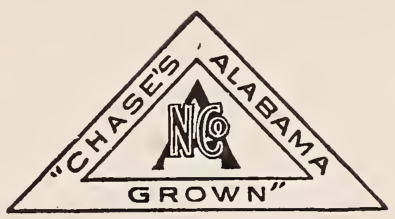

\section{BOX LOTS FOR SALE}

As packed; to be shipped after February I8th, when wanted, from St. Louis, Rochester, and Philadelphia. Condition guaranteed.

You've got sense and know us; we're too busy to talk. Figure it out and send orders to

\section{Alabama Nursery Company HUNTSVILLE, ALABAMA}

One Mule barometer free with each order, if you ask for it. 


\section{ON BOARD CARS ST. LOUIS}

\section{FRUITS, ASSORTED}

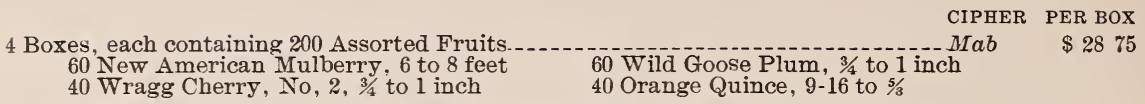

These Wragg are No. 2 because some trees are branched two low, some are not smooth, all are WELL ROOTED.

\section{$\mathrm{PEACH}$}

\section{5-8 TO 3-4, 5 TO 6 FEET}

4 Boxes, each containing 210 Assorted 40 Alexander 50 Hiley

60 Sneed

70 Greensboro

Deacon

$\$ 1400$

2 Boxes, each containing 210 Assorted

40 Alexander
50 Hiley

60 Sneed

20 Triump

10 Bokara

10 Triumph

1 Box containing 276 Assorted 176 Amsden 100

20 Triumph Deaden 170 Hiley 70 Gov. Hogg

50 Sneed

40 Blood Cling

$20 \mathrm{Hiley}$

Deafen

50 Early Tillotson

60 Amsden

30 Greensboro

9-16 TO 5-8, 4 TO 5 FEET

4 Boxes, each containing 340 Assorted

20 Alexander

20 Bokara

30 Fam. Favorite

40 Gov. Hogg
60 Greensboro

20 Mamie Ross

Diacid

1750

2 Boxes, each containing 300 Assorte 60 Amsden 210 Hiley

2 Boxes, each containing 300 Hiley

1 Box containing 247 Assorted. 10 Fam. Farorite

70 Blood Cling 30 Bokara

20 Amsden

in Gov. Hogg

7 Mamie Ross
40 Sneed

100 Hiley

10 Wheatland

\section{TO 3 FEET}

7 Boxes, each containing 775 Assnrted 50 Crawfords Early

75 Foster 2100 Alexander 100 Bokara Dwarf

1500

GOV. HOGG;-First cling to ripen, one of the largest, excellent quality.

HILEY; - White with red cheek, very large, free. Two weeks earlier than Elberta, best shipping sort of its season.

\section{PLUM ON MARIANNA}

1 Box containing 200 Burbank, 5 to 6 feet, strong whips Hub

These are 1 year old, clean and A 1 . You cannot find better trees.

\section{CURRANTS}

1 Box containing 538 Currants and Gooseberries, 2 year, No. 525 Red Dutch Currant

13 Downing Gooseberry Made

1 Box containing $55_{0}$ Red Dutch, 1 year, No. 1 ... 


\section{CALIFORNIA PRIVET}

CIPHER PER BOX

7 Boxes, each containing 1000 California Privet, 1 year, 8 to 18 inches, transp. size Tiara $\$ 750$ 8 Boxes. each containing 1000 California Privet, 2 year, 12 to 18 inches, lightly br._-_Tibba 1000 6 Boxes, each containing 500 California Privet, 2 year, 18 to 24 inches. well br.....Tibia 1100 3 Boxes, each containing 1000 California Privet, 2 year, 18 to 24 inches, well br.-...Tical 2200 6 Boxes, each containing 500 California Privet, 2 year, 2 to 3 feet, well br______tick 1550 3 Boxes, each containing 500 California Privet, 2 jear, 3 to 4 feet, lightly br._-_.Ticker 1350 3 Boxes, each containing 600 California Privet, 2 year, 3 to 4 feet, lightly br......-Tibet 1500

\section{HARDY ROSES}

\section{ALABAMA GROWN, OWN ROOTS}

$\mathrm{XX}$ size extra heavy, cut back to 30 inches of wood.

11 Boxes, each containing 100 Crimson Rambler -

2 Boxes, each containing 150 Magna Charta . Tablet

750

4 Boxes, each containing 200 Crimson Rambler-..-_-

No. 1 size, strong plants, cut back to 24 inches of wood.

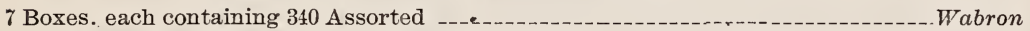

100 Baltimore Belle

70 Madam Plantier

70 Queen of Prairie 100 Crimson Rambler

3 Boxes, each containing 160 Magna Charta

3 Boxes, each containing 100 Crimson Rambler

1100

5 Boxes, each containing 300 Crimson Rambler,

1 Box containing 250 Assorted Roses, No. 1

50 Madam Plantier

100 Queen of Prairie

Wad

50 Baltimore Belle

50 Crimson Ramble

2100

1275

IMPORTED-BUDDED ON MANETTI, First Size and Grade.

4 Boxes, each containing 100 Assorted

10 Harrison's Yellow

20 Ulrich Brunner

10 Madam Plantier

\section{ALABAMA GROWN}

No. $1 \frac{1}{2}$ size, not quite large enough for No. 1 plants, have 18 inches of wood, 3 or more branches.

1 Box containing 320 Magna Charta

14 Boxes, each containing 300 Assorted. 100 Queen of Prairie

100 Madam Plantier

100 Crimson Rambler

20 Crimson Rambler

Waddle

1000

19 Boxes, each containing 250 Crimson Rambler Tabby

1400

1025

14 Boxes, each containing 350 Crimson Rambler

Table

1125

1575

CRIMSON RAMBLER for tranplauting, light 1 year plants, with 12 inches of wood.

6 Boxes, each containing 500 Crimson Rambler

7 Boxes, each containing 350 Crimson Rambler Wadder 1050

1 Box containing 550 Crimson Rambler

Wade 740

Waffle 1150

\section{ASSORTED SHRUBS AND ROSES}

1 Box containing 1078 Assorted, 2 to 3 feet 1 to 2 feet 6 to 12 ins.

Wiegelia, Variegated....-
Deutzia, Pride of Roch..--

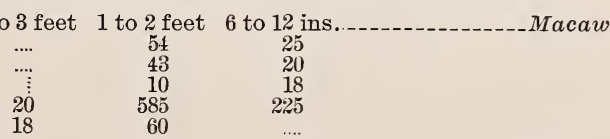

1900

2 Boxes, each containing 630 Assorted

200 Hydrangea Layers, 12 to 18 inches

210 Snowball, 12 to 18 inches

2 Boxes, each containing 280 Assorted -

35 Spirea Van Houtte, 3 to 4 feet, well br. 40 Althea. Grand. Superba, (dble.white and red)

5 Deutzia, Pride of Roch. 3-4 ft. Well br.

40 Forsythia Sieboldi, 3 to 4 feet, well br.

40 Crimson Rambler XंX

50 Queen of Proirie XX

3 to 4 feet, well branched
thea, Jeanne de Arc, finest, 3-4 ft. well br. (double pure white)

30 Japan Golden Honeysuckle No. 1

2 Boxes, each containing 330 Assorted

20 Deutzia P. of Rochester, 2 to $3 \mathrm{ft}$. 2 yr. 30 Japan Golden Honeysuckle No. 1

90 Spirea Van Houtte, 2 to 3 feet, 2 year $\quad 70$ Crimson Rambler No. $11 / 2$

50 Forsythia Fortunei, 2 to 3 feet, 2 year $\quad 70$ Queen of Prairie No. $1 \frac{1}{2}$ 


\section{ON BOARD CARS ROCHESTER ROSES}

1 Box containing 100 Dorothy Perkins XX $r \begin{array}{r}\text { CIPHER PER BOX } \\ \$ 750\end{array}$

\section{ON BOARD CARS PHILADELPHIA FLOWERING ALMOND, PRUNUS TRILOBA}

CIPHER PER BOX

1 Box containing 400 Assorted, on plum roots 100 Flowering Almond, (dble. white) 1-2 ft. 130 Prunus Triloba, 3 to 4 feet

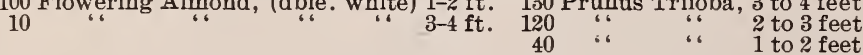

\section{ROSES}

3 Boxes, each containing $750 \mathrm{~W}$ ichurianna No. 2 Wader

These plants have all been cut back to 18 inches of wood.

A9 Address all orders for Stock shown in this List to ALABAMA NURSERY COMPANY. Huntsville, Alabama. 